\title{
Atomic detection in microwave cavity experiments: a dynamical model
}

\author{
R. Rossi Jr. and M. C. Nemes \\ Departamento de Física, Instituto de Ciências Exatas, \\ Universidade Federal de Minas Gerais, C.P. 702, \\ 30161-970, Belo Horizonte, MG, Brazil \\ J. G. Peixoto de Faria \\ Departamento Acadêmico de Ciências Básicas, \\ Centro Federal de Educação Tecnológica de Minas Gerais, \\ 30510-000, Belo Horizonte, MG, Brazil
}

\begin{abstract}
We construct a model for the detection of one atom maser in the context of cavity Quantum Electrodynamics (QED) used to study coherence properties of superpositions of electromagnetic modes. Analytic expressions for the atomic ionization are obtained, considering the imperfections of the measurement process due to the probabilistic nature of the interactions between the ionization field and the atoms. Limited efficiency and false counting rates are considered in a dynamical context, and consequent results on the information about the state of the cavity modes are obtained.
\end{abstract}

PACS numbers: 42.50.Pq, 32.50.Rm, 07.77.Gx

Keywords: Cavity quantum electrodynamics, Field ionization detector 


\section{INTRODUCTION}

The quantum interaction between two level Rydberg atoms and one microwave mode inside a high quality factor $(Q)$ cavity has been crucial for our understanding of dissipation and decoherence in quantum mechanics $[1,2]$. Usually in cavity quantum electrodynamics (QED) experiments, Rydberg atoms cross an experimental array constituted of two Ramsey zones and a high $Q$ cavity. Thereafter their final state is detected in two stages. Firstly, they cross an electromagnetic field which is built to ionize the highest state of the atom. The second detection zone is designed to detect the lower atomic level.

Most of the work available in literature about the detection process is based on statistical assumptions. The pumped atoms are statistically independent, so that their arrival times are subject to a Poissonian or other statistics [3-5]. The basic idea is that atoms arrive at random and they are recorded at equally random times, so the only reproducible data are statistical. In this context one is lead to studying the statistic of detector clicks. There are numerical studies [6, 7] and also analytical results by Rempe and Walther [8] and also by Paul and Richter [9].

In the present contribution we propose (to our knowledge for the first time) a dynamical model for the detection process. We assume that the atom undergoes the influence of a classical electrical field when it traverses the detection zones. The net effect of this field is to couple one (in the case of intrinsically inefficient detectors) or two (in the case of detectors that register false countings) discrete atomic levels to the continuum. If the atom is ionized, a transition to the continuum has ocurred and a classical signal - a "click" - is generated in the correspondent detector. However, if the atom remains in one of the two discrete levels, no click is registered by the detector.

Since the atom works as a probe to the field stored in a high- $Q$ cavity, the click or no-click registered by the detectors represents a gain of information about the state of the compound system formed by the atom and by the high- $Q$ cavity field. Hence, the process of detection can be divided in two parts. First, the state of the compound system atom-high- $Q$ cavity field undergoes an unitary evolution during the passage of the atom through each detection

zone. Next, the resultant state is projected into a proper subspace defined as follows: if a click is registered, this subspace is formed by the set of the states that form the continuum; otherwise, this subspace corresponds to the states associated to the two discrete levels. 
This paper is organized in the following way. In Sec. II, we treat a model for intrinsically inefficient detectors. The analytical form of our results are exactly the same as those in reference [10], whose derivation is based on statistical and physically plausible arguments. In Sec. III, we study the possibility of the detectors perform false countings. In this case, we found that the probability of a click depends on the "non-diagonal" terms of the state of the system atom-high-Q cavity field. We calculate the fidelity of the field states in high- $Q$ cavity after the measurement process considering the two kinds of imperfections, limited efficiency and false detections. Sec. IV contains a summary of the results and conclusions.

\section{MODEL FOR INEFFICIENT DETECTOR}

The ionization process of an atom due to its interaction with an electromagnetic field is considered in a quantum context. Therefore a finite probability of non-excitation will exist. That is what we call an intrinsic (i.e., quantum mechanical) inefficiency.

The Hamiltonian which describes the interaction between two level atoms and ionization field on the first detection zone, and takes into account only the intrinsic inefficiency of the process is given by (the hamiltonian for the second detection zone can been obtained replacing the index $e$ by $g$ ):

$$
H_{1 e}=\epsilon_{e}|e\rangle\left\langle e\left|+\epsilon_{g}\right| g\right\rangle\left\langle g\left|+\int d k \epsilon_{k}\right| k\right\rangle\langle k|+v_{e} \int d k(|e\rangle\langle k|+| k\rangle\langle e|) .
$$

The first and second terms in the Hamiltonian stand for the two discrete atomic levels $|e\rangle$ and $|g\rangle$, excited and ground state respectively, with energies $\epsilon_{e}$ and $\epsilon_{g}$. The third term represents its continuum spectrum. The last term accounts for the coupling between the highest discrete level and the continuum. The strength of this interaction is given by the parameter $v_{e}$, assumed state independent for simplicity. This term is responsible for the ionization of the atom. Since we are dealing with a quantum mechanical process, which is intrinsically probabilistic, we will also have to consider the possibility of non-ionization of the atom.

Following Cohen [11], the evolution of the discrete state $|e\rangle$ according to (1) is given by:

$$
|\psi(t)\rangle=\mathrm{e}^{-i H_{1 e} t / \hbar}|e\rangle=\int d \mu\left\langle\psi_{\mu}^{e} \mid e\right\rangle \mathrm{e}^{-i \epsilon_{\mu}^{e} t / \hbar}\left|\psi_{\mu}\right\rangle,
$$

where $\left\{\left|\psi_{\mu}^{e}\right\rangle\right\}$ and $\left\{\epsilon_{\mu}^{e}\right\}$ correspond to the set of eigenvectors and eigenvalues of $H_{1 e}$ respec- 
tively. The coefficients $\left\langle\psi_{\mu}^{e} \mid e\right\rangle$ and $\left\langle\psi_{\mu}^{e} \mid k\right\rangle$ may be written as

$$
\begin{aligned}
\left\langle\psi_{\mu}^{e} \mid e\right\rangle & =\frac{1}{\left[1+\int \mathrm{d} k^{\prime}\left(\frac{v}{\epsilon_{\mu}^{e}-\epsilon_{k^{\prime}}}\right)^{2}\right]^{1 / 2}}, \\
\left\langle\psi_{\mu}^{e} \mid k\right\rangle & =\frac{v /\left(\epsilon_{\mu}^{e}-\epsilon_{k}\right)}{\left[1+\int \mathrm{d} k^{\prime}\left(\frac{v}{\epsilon_{\mu}^{e}-\epsilon_{k^{\prime}}}\right)^{2}\right]^{1 / 2}} .
\end{aligned}
$$

Accordingly, the ionization probability can be obtained as follows

$$
\begin{aligned}
p_{e} & =\int \mathrm{d} k|\langle k \mid \psi(t)\rangle|^{2} \\
& =\int \mathrm{d} k\left|\int \mathrm{d} \mu\left\langle\psi_{\mu}^{e} \mid e\right\rangle\left\langle k \mid \psi_{\mu}^{e}\right\rangle \mathrm{e}^{-i \epsilon_{\mu}^{e} t / \hbar}\right|^{2} .
\end{aligned}
$$

This probability defines the detector's efficiency. Therefore the non-detection probability is given by $1-p_{e}=\left.\left.\left|\int \mathrm{d} \mu \mathrm{e}^{-i \epsilon_{\mu}^{e} t / \hbar}\right|\left\langle\psi_{\mu}^{e} \mid e\right\rangle\right|^{2}\right|^{2}$. After some simplifications (see [11]) the nondetection probability can be written as

$$
1-p_{e}=\mathrm{e}^{-\Gamma|t|}
$$

where $\Gamma$ is the transition rate from discrete to the continuum level, calculated from Fermi's golden rule. $\Gamma$ is given by

$$
\Gamma=\frac{2 \pi \rho(E)}{\hbar}
$$

where $\rho(E)$ is the level density per unity energy. In the limit where the atom ionization time can be considered to be infinite (in some experimental context) we will have a perfect detector.

Following the same procedure for $H_{1 g}$ we find $p_{g}=\int \mathrm{d} k\left|\int \mathrm{d} \mu\left\langle\psi_{\mu}^{g} \mid g\right\rangle\left\langle k \mid \psi_{\mu}^{g}\right\rangle \mathrm{e}^{-i \epsilon_{\mu}^{g} t / \hbar}\right|^{2}$, where $\left\{\left|\psi_{\mu}^{g}\right\rangle\right\}$ and $\left\{\epsilon_{\mu}^{g}\right\}$ correspond to the set of eigenvectors and eigenvalues of $H_{1 g}$ respectively.

\section{A. An exemple: cavity QED}

As an exemple of applicability of the model, let us study the interactions between two level atoms and their detection through ionization fields in cavity QED experiments. The state of the system atom-high- $Q$ cavity field can be written as

$$
\rho_{A C}(0)=\rho_{e e}|e\rangle\left\langle e\left|+\rho_{e g}\right| e\right\rangle\left\langle g\left|+\rho_{g e}\right| g\right\rangle\left\langle e\left|+\rho_{g g}\right| g\right\rangle\langle g| \text {. }
$$




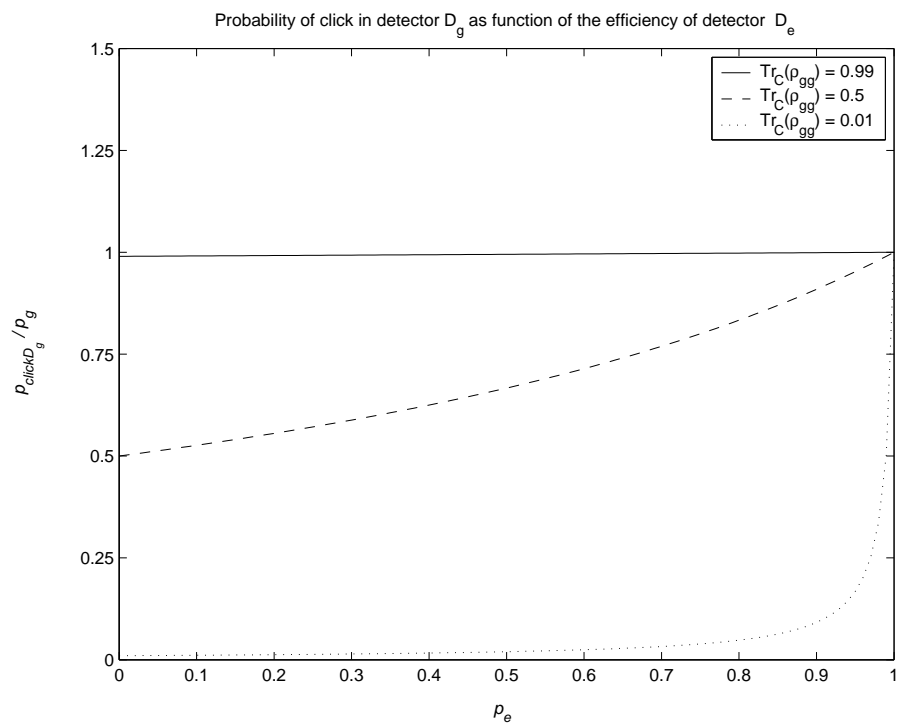

FIG. 1: Influence of the efficiency of the detector $D_{e}\left(p_{e}\right)$ on the "normalized" probability of click in the detector $D_{g}\left(p_{\text {click } D_{g}} / p_{g}\right)$, for different values of $\operatorname{Tr}_{C}\left(\rho_{g g}\right)$. The efficiency of the detector $D_{g}$ $\left(p_{g}\right)$ just limits the maximum value reached by $p_{\operatorname{click} D_{g}}$ and does not modify its qualitative behavior as function of $p_{e}$.

This state represents the most general state (in the system atom-cavity field) immediately before the interaction between the atom and the detectors. The symbols $\rho_{e e}, \rho_{e g}, \rho_{g e}$ and $\rho_{g g}$ are operators in the cavity field subsystem.

The interaction between the atom and the first detection zone $\left(D_{e}\right)$ can be separated in two steps. Firstly, a quantum unitary evolution governed by the Hamiltonian $H_{1 e}$ given by Eq. (1) during the time interval $t_{1}$. The atom-cavity field state, after this process, is given by

$$
\rho_{A C}\left(t_{1}\right)=\mathrm{e}^{-i H_{1 e} t_{1} / \hbar} \rho_{A C}(0) \mathrm{e}^{i H_{1 e} t_{1} / \hbar}
$$

Now, in the second step, at the time $t_{1}$, a classical signal is generated. If the detector clicks, we will know that the atom was ionized, so $\rho_{A C}\left(t_{1}\right)$ must be projected into the subspace $\{|k\rangle\}$. Although, if $D_{e}$ does not click we know that the atomic state must be projected into subspace spanned by the discrete levels $\{|e\rangle,|g\rangle\}$. The maximum value that $t_{1}$ can assume is $t_{1}^{\prime}$ which is the time taken by the atom to cross $D_{e}$ completely. So, up to $t_{1}^{\prime}$, we will certainly acquire information about the system. This revealed information plays an essencial role into $\rho_{A C}$ 's evolution. So we are aware that before the interaction with $D_{g}$, the state $\rho_{A C}\left(t_{1}\right)$ must be projected into properly subspace. 
We can calculate the probability of a click in $D_{e}$

$$
p_{\text {click } D_{e}}=\int \mathrm{d} k \operatorname{Tr}\left(|k\rangle\langle k| \rho_{A C}\left(t_{1}\right)\right)=\int \mathrm{d} k \operatorname{Tr}\left(|k\rangle\left\langle k\left|\rho_{e e} \mathrm{e}^{-i H_{1 e} t_{1} / \hbar}\right| e\right\rangle\langle e| \mathrm{e}^{i H_{1 e} t_{1} / \hbar}\right) .
$$

Then, using Eq. (4), we may write

$$
p_{\text {click } D_{e}}=p_{e} \operatorname{Tr}_{C}\left(\rho_{e e}\right)
$$

where $\operatorname{Tr}_{C}$ is the partial trace on the cavity-field subspace. This product can be interpreted as the efficiency of $D_{e}\left(p_{e}\right)$ times the probability of click on a perfect detector after the interaction with the state $\rho_{A C}(0)\left(\operatorname{Tr}_{C}\left(\rho_{e e}\right)\right)$.

The non-click probability is:

$$
p_{\text {non-click } D_{e}}=\operatorname{Tr}\left[(|e\rangle\langle e|+| g\rangle\langle g|) \rho_{A C}\left(t_{1}\right)\right]=\operatorname{Tr}_{C}\left(\rho_{e e}\right)+\operatorname{Tr}_{C}\left(\rho_{g g}\right)-p_{e} \operatorname{Tr}_{C}\left(\rho_{e e}\right) .
$$

From the normalization of $\rho_{A C}(0), \operatorname{Tr}_{C}\left(\rho_{e e}\right)+\operatorname{Tr}_{C}\left(\rho_{g g}\right)=1$, we can write

$$
p_{\text {non-click } D_{e}}=1-p_{e} \operatorname{Tr}_{C}\left(\rho_{e e}\right) .
$$

After the non-click stage the system is in the state:

$$
\begin{aligned}
\rho_{\text {non-click }}\left(t_{1}\right) & =\frac{(|e\rangle\langle e|+| g\rangle\langle g|) \rho_{A C}\left(t_{1}\right)(|e\rangle\langle e|+| g\rangle\langle g|)}{N} \\
& =\frac{\rho_{g g}|g\rangle\left\langle g\left|+\rho_{e e}\left(1-p_{e}\right)\right| e\right\rangle\langle e|+\left(\rho_{e g} \mathrm{e}^{i \epsilon_{g} t_{1} / \hbar} \int \mathrm{d} \mu e^{-i \epsilon_{\mu} t_{1} / \hbar}\left|\left\langle\psi_{\mu} \mid e\right\rangle\right|^{2}|e\rangle\langle g|+h . c .\right)}{N},
\end{aligned}
$$

where $N=1-p_{e} \operatorname{Tr}_{c} \rho_{e e}$. Note that if the efficiency is equal to unity $\left(p_{e}=1\right)$, the reduced state operator on atomic subspace can be written as $\rho_{g g}|g\rangle\langle g|$. This result was expected, as we know, for perfect detectors a non-click in $D_{e}$ would lead to the projection $|g\rangle\left\langle g\left|\rho_{A C}(0)\right| g\right\rangle\langle g|$.

When the atom is not ionized on $D_{e}$, it continues the journey and passes through the second detection zone $\left(D_{g}\right)$. Let us set the interaction time between atom and the electromagnetic field inside $D_{g}$ by $t_{2}$. The temporal evolution that models this interaction is again unitary:

$$
\rho_{A C}\left(t_{2}\right)=\frac{1}{N} \mathrm{e}^{-i H_{1 g}\left(t_{2}-t_{1}\right) \hbar} \rho_{\text {non-click }}\left(t_{1}\right) \mathrm{e}^{i H_{1 g}\left(t_{2}-t_{1}\right) \hbar} .
$$

So, the probability of click in $D_{g}$ is

$$
p_{\text {click } D_{g}}=\frac{p_{g} \operatorname{Tr}_{C}\left(\rho_{g g}\right)}{1-p_{e} \operatorname{Tr}_{C}\left(\rho_{e e}\right)}
$$


Note that this probability depends on the efficiency of the first detector $\left(p_{e}\right)$. Now let us examine some limits. For $p_{e}=0$, this is equivalent to the situation where the first detector is absent, so the atom interacts just with the second ionization zone $D_{g}$ and the probability of click is $p_{g} \operatorname{Tr}_{C}\left(\rho_{g g}\right)$, as expected. If $p_{e}=1$, the first detector is perfect, therefore we know that when an atom crosses $D_{e}$ without been detected, the system goes to the state $|g\rangle$, as discussed before, and the probability of click is the efficiency of $D_{g}\left(p_{g}\right)$. If both detectors are perfect $\left(p_{e}=p_{g}=1\right), p_{\text {click } D_{g}}=1$ because the second detector will not miss any atom prepared in $|g\rangle$.

A more complete analysis of $p_{\text {click } D_{g}}$ for different $\rho_{A C}(0)$ is shown in Fig. 1. The behavior of the curves associate to $\operatorname{Tr}_{C}\left(\rho_{g g}\right)=0.5$ and $\operatorname{Tr}_{C}\left(\rho_{g g}\right)=0.01$ reflects the fact that a non-click on a very efficient $D_{e}\left(p_{e} \approx 1\right)$ raises the probability $p_{\text {click } D_{g}}$, even if the atom is practically prepared in the state $|e\rangle\langle e|\left(\operatorname{Tr}_{C}\left(\rho_{g g}\right)=0.01\right)$. On the other hand, if the atom is practically prepared in the state $|g\rangle\langle g|\left(\operatorname{Tr}_{C}\left(\rho_{g g}\right)=0.99\right), p_{e}$ does not affect $p_{\text {click } D_{g}}$.

The probability of non-click in $D_{g}$ is

$$
1-p_{\text {click } D_{g}}=\frac{1-p_{e} \operatorname{Tr}_{C} \rho_{e e}-p_{g} \operatorname{Tr}_{C} \rho_{g g}}{1-p_{e} \operatorname{Tr}_{C} \rho_{e e}}
$$

When the atom crosses both detectors without being detected, it reduces the field state inside the cavity to

$$
\rho_{C}^{\prime}=\frac{\operatorname{Tr}_{A}\left[(|e\rangle\langle e|+| g\rangle\langle g|) \rho_{A C}\left(t_{2}-t_{1}\right)(|e\rangle\langle e|+| g\rangle\langle g|)\right]}{\operatorname{Tr}\left[(|e\rangle\langle e|+| g\rangle\langle g|) \rho_{A C}\left(t_{2}-t_{1}\right)(|e\rangle\langle e|+| g\rangle\langle g|)\right]} .
$$

Here, $\operatorname{Tr}_{A}$ stands for the trace in the atomic variables. Now, using the definition (4) we may write:

$$
\rho_{C}^{\prime}=\frac{\left(1-p_{e}\right) \rho_{e e}+\left(1-p_{g}\right) \rho_{g g}}{1-p_{e} \operatorname{Tr}_{C}\left(\rho_{e e}\right)-p_{g} \operatorname{Tr}_{C}\left(\rho_{g g}\right)} .
$$

The form of this result is in complete agreement with the one in [10], where the authors used statistics arguments to derive the expression (18).

\section{MODEL FOR FALSE DETECTIONS}

In addition to the previous intrinsically inefficient detector we extend the model to include false detections. The hamiltonian for the first detection zone $D_{e}$ is given by (the hamiltonian 
for the second detection zone can been obtained replacing the index $e$ by $g$ ):

$$
\begin{aligned}
H_{2 e} & =\epsilon_{e}|e\rangle\left\langle e\left|+\epsilon_{g}\right| g\right\rangle\left\langle g\left|+\int \mathrm{d} k \epsilon_{k}\right| k\right\rangle\langle k| \\
& +w_{e} \int \mathrm{d} k(|e\rangle\langle k|+| k\rangle\langle e|)+w_{g} \int \mathrm{d} k(|g\rangle\langle k|+| k\rangle\langle g|),
\end{aligned}
$$

where $w_{e}$ and $w_{g}$ are real coupling constants. The second interaction term (the last one in the equation above) represents the coupling between $|g\rangle$ and the continuum, so it is responsible for wrong detections. On the other hand, the previous one is responsible for the correct ones.

For simplicity we are going to define the complex coefficient

$$
q_{a, b}^{e}=\int \mathrm{d} \eta \mathrm{e}^{-i \epsilon_{\eta}^{e} t / \hbar}\left\langle\phi_{\eta}^{e} \mid a\right\rangle\left\langle b \mid \phi_{\eta}^{e}\right\rangle
$$

where $\left\{\left|\phi_{\eta}^{e}\right\rangle\right\}$ and $\left\{\epsilon_{\eta}^{e}\right\}$ are the set of eigenvectors and eigenvalues of $H_{2 e}$ respectively. $a$ and $b$ are indexes that may represent continuum and discrete eigenvectors. The explicit form of the coefficients inside the integral and relative discussions are in the appendix. The notation is as follows: the upper index indicates which detection zone the atom is passing through. The two lower indexes, $a$ and $b$, represent its initial incoming state and its final state after traversing the detector, respectively. One can notice that $\int \mathrm{d} k\left|q_{e, k}^{e}\right|^{2}$ is the probability of an atom prepared in $|e\rangle$ to be ionized inside $D_{e}$, this can be understood as the efficiency of $D_{e} \cdot \int \mathrm{d} k\left|q_{g, k}^{e}\right|^{2}$ is the probability of an atom prepared in $|g\rangle$ to be ionized inside $D_{e}$, i.e., the probability of a wrong detection.

We can see, from (19), that the unitary evolution of this system allows for an indirect coupling between the two discrete levels. So we can take into account $\left|q_{e, g}^{e}\right|^{2}$ which is the probability of a transition between the two discrete levels. $\left|q_{e, e}^{e}\right|^{2}\left(\left|q_{g, g}^{e}\right|^{2}\right)$ is the probability of an atom prepared in $|e\rangle(|g\rangle)$ to interact with the electromagnetic field inside $D_{e}$ and do not change level. We can also notice that $\int \mathrm{d} k\left|q_{e, k}^{g}\right|^{2}\left(\int \mathrm{d} k\left|q_{g, k}^{g}\right|^{2}\right)$ is the probability that an atom prepared in $|e\rangle(|g\rangle)$ to be ionized inside $D_{g}$.

\section{A. An example: cavity QED}

As we did for the intrinsically inefficient detectors, the interaction between atoms and false counting detectors can be separated in two processes: firstly, an unitary evolution of the initial state operator, generated by $H_{2 e}\left(H_{2 g}\right)$ where $H_{2 e}\left(H_{2 g}\right)$ have the form shown in 
Eq. (19), and then a projection in a subspace, which represents the classical information, click or non-click, on the detector.

Starting from the initial state given by Eq. (7), and using the definitions in Eq. (20), the probability of click in $D_{e}$ can be written as

$$
p_{\text {click } D_{e}}=\int \mathrm{d} k\left|q_{e, k}^{e}\right|^{2} \operatorname{Tr}_{C}\left(\rho_{e e}\right)+\int d k\left|q_{g, k}^{e}\right|^{2} \operatorname{Tr}_{C}\left(\rho_{g g}\right)+\left(\int \mathrm{d} k q_{e, k}^{e *} q_{g, k}^{e} \operatorname{Tr}_{C}\left(\rho_{e g}\right)+\text { h.c. }\right) .
$$

This expression shows us that $p_{\text {click } D_{e}}$ is sensitive to interference terms $\left(\rho_{e g}\right)$ and $\left(\rho_{g e}\right)$. If we calculate the value of $p_{\text {click } D_{e}}$ for the initial state $\rho_{A C}(0)=\rho_{e e}|e\rangle\left\langle e\left|+\rho_{g g}\right| g\right\rangle\langle g|$, the answer would be different from (21). However, if we do the same, but replacing the false counting detectors by inefficient or perfect detectors, the calculated probability would be the same for the two different initial states. That is due to the fact that this case is insensitive to interference terms.

In order to compare the modifications on the cavity field due to atomic interaction with inefficient detectors and false counting detectors, we calculate the fidelity of the different state operators. Fidelity between the states $\rho_{A}$ and $\rho_{B}$ measures the overlap between them and is given by

$$
F\left(\rho_{A}, \rho_{B}\right)=\left(\operatorname{Tr} \sqrt{\rho_{A}^{1 / 2} \rho_{B} \rho_{A}^{1 / 2}}\right)^{2} .
$$

Firstly, let us calcule the fidelity between state operators $\rho_{A}^{e}$ (which describe the system after the atomic ionization inside the first detection zone of inefficient detectors), and $\rho_{B}^{e}$ (which describe the system after the atomic ionization inside the first detection zone of false counting detectors). For simplicity, assume that the system atom-high- $Q$ cavity field is found in the following entangled state just before the atom reaches the detection zones:

$$
\rho_{A C}(0)=\frac{1}{2}(|e, 0\rangle\langle e, 0|+| e, 0\rangle\langle g, 1|+| g, 1\rangle\langle e, 0|+| g, 1\rangle\langle g, 1|) .
$$

After an unitary evolution and the projection on the continuum subspace, $\rho_{A}^{e}$ and $\rho_{B}^{e}$ can be written as

$$
\begin{aligned}
& \rho_{A}^{e}=|0\rangle\langle 0|, \\
& \rho_{B}^{e}=\frac{\int \mathrm{d} k\left(\left|q_{e, k}^{e}\right|^{2}|0\rangle\left\langle\left. 0|+| q_{g, k}^{e}\right|^{2} \mid 1\right\rangle\left\langle 1\left|+q_{g, k}^{e *} q_{e, k}^{e}\right| 1\right\rangle\left\langle 0\left|+q_{e, k}^{e *} q_{g, k}^{e}\right| 0\right\rangle\langle 1|\right)}{\int \mathrm{d} k\left(\left|q_{e, k}^{e}\right|^{2}+\left|q_{g, k}^{e}\right|^{2}\right)},
\end{aligned}
$$

and the fidelity

$$
F\left(\rho_{A}^{e}, \rho_{B}^{e}\right)=\frac{\int \mathrm{d} k\left|q_{e, k}^{e}\right|^{2}}{\int \mathrm{d} k\left(\left|q_{e, k}^{e}\right|^{2}+\left|q_{g, k}^{e}\right|^{2}\right)} .
$$


Notice that if the wrong detection probability goes to zero $\left(\int d k\left|q_{g, k}^{e}\right|^{2} \rightarrow 0\right)$, the fidelity goes to one, $F\left(\rho_{A}^{e}, \rho_{B}^{e}\right) \rightarrow 1$, so $\rho_{A}^{e}$ and $\rho_{B}^{e}$ became identical.

Now, we are going to calcule the fidelity between state operators $\rho_{A}^{g}$, which describes the system after the atomic ionization inside the second detection zone of inefficient detectors, and $\rho_{B}^{g}$, which describes the system the atomic ionization inside the second detection zone of false counting detectors. The calculation follows as this: interaction of atom with the first detection zone $D_{e}$, modeled by unitary evolution of the state given by Eq. (23) and projection on the discrete subspace. Then, the interaction with the second detection zone $D_{g}$, modeled again by unitary evolution of the resultant state and projection, but now on the continuum subspace. After this, we can write the fidelity as

$$
F\left(\rho_{A}^{g}, \rho_{B}^{g}\right)=\frac{1}{A} \int \mathrm{d} k\left(\left|q_{g, g}^{e *}\right|^{2}\left|q_{g, k}^{g *}\right|^{2}+q_{g, g}^{e *} q_{g, e}^{e} q_{g, k}^{g *} q_{e, k}^{g}+q_{g, g}^{e} q_{g, e}^{e *} q_{g, k}^{g} q_{e, k}^{g *}+\left|q_{g, e}^{e}\right|^{2}\left|q_{e, k}^{g}\right|^{2}\right)
$$

where

$$
\begin{aligned}
A & =\int \mathrm{d} k\left(\left|q_{g, e}^{e}\right|^{2}\left|q_{g, k}^{g}\right|^{2}+q_{g, e}^{e *} q_{e, e}^{e} q_{g, k}^{g *} q_{e, k}^{g}+q_{g, e}^{e} q_{e, e}^{e *} q_{g, k}^{g} q_{e, k}^{g *}+\left|q_{e, e}^{e}\right|^{2}\left|q_{e, k}^{g}\right|^{2}\right) \\
& +\int \mathrm{d} k\left(\left|q_{g, g}^{e}\right|^{2}\left|q_{g, k}^{g}\right|^{2}+q_{g, g}^{e *} q_{g, e}^{e} q_{g, k}^{g *} q_{e, k}^{g}+q_{g, g}^{e} q_{g, e}^{e *} q_{g, k}^{g} q_{e, k}^{g *}+\left|q_{g, e}^{e}\right|^{2}\left|q_{e, k}^{g}\right|^{2}\right)
\end{aligned}
$$

As we are considering that any transition from a discrete state to the continuum generates a classical signal, we must not admit the possibility of indirect coupling between $|e\rangle$ and $|g\rangle$ mediated by the continuum. Therefore, we must assume that $\left|q_{g, e}^{e}\right|^{2}=0$ and we may write:

$$
F\left(\rho_{A}^{g}, \rho_{B}^{g}\right)=\frac{\int \mathrm{d} k\left|q_{g, g}^{e}\right|^{2}\left|q_{g, k}^{g}\right|^{2}}{\int \mathrm{d} k\left(\left|q_{g, g}^{e}\right|^{2}\left|q_{g, k}^{g}\right|^{2}+\left|q_{e, e}^{e}\right|^{2}\left|q_{e, k}^{g}\right|^{2}\right)} .
$$

If the wrong detections probability in $D_{g}$ goes to zero $\left(\int d k\left|q_{e, k}^{g}\right|^{2} \rightarrow 0\right)$ the fidelity goes to one $\left[F\left(\rho_{A}^{g}, \rho_{B}^{g}\right) \rightarrow 1\right]$.

\section{CONCLUSIONS}

We have presented a dynamical model for the detection process of atomic levels on field ionization detectors. On the context of cavity QED, the model allows us to calculate the reduced state operator, for the field inside the cavity, after the classical signal generated by the detectors.

The detailed analysis of the detection process also let us introduce naturally the effects of realistic features of the detectores (e.g. efficiency and false counting rates) on the study 
of microwave cavity experiments. For intrinsically inefficient detectors, we found that the probability of a click in the second detection zone is sensitive to the efficiency of the first one. Besides, our results are in complete agreement with those obtained in Ref. [10] by different methods.

If one allows the detectors to register false countings, the probability of a click is sensitive to the "non-diagonal" or coherence terms of the state of the system atom-high- $Q$ cavity field. In fact, false countings are a consequence of the coupling between the two discrete atomic levels to the continuum in each detection zone. As a result of this coupling, a click registered in any detector does not provide an unequivocal information about the atomic state. The detectors acts as a "beam splitter", mixing the two "paths" $e$ and $g$, and, to some degree, these "paths" become undistinguishable.

\section{APPENDIX: EVALUATION OF THE COEFFICIENTS $\left\langle a \mid \phi_{\eta}^{e}\right\rangle$}

In order to calculate the coefficients inside the integral in (20), let us write the eigenvalue equation for $H_{2 e}$ :

$$
H_{2 e}\left|\phi_{\eta}^{e}\right\rangle=\left[H_{e(0)}+H_{2 e(I)}+H_{2 e(I I)}\right]\left|\phi_{\eta}^{e}\right\rangle=\epsilon_{\eta}^{e}\left|\phi_{\eta}^{e}\right\rangle
$$

where $\left|\phi_{\eta}^{e}\right\rangle$ and $\epsilon_{\eta}^{e}$ are eigenvectors and eigenvalues of the Hamiltonian $H_{2 e}, H_{e(0)}=\epsilon_{e}|e\rangle\langle e|+$ $\epsilon_{g}|g\rangle\left\langle g\left|+\int \mathrm{d} k \epsilon_{k}\right| k\right\rangle\langle k|, H_{2 e(I)}=w_{e} \int \mathrm{d} k(|e\rangle\langle k|+| k\rangle\langle e|)$ and $H_{2 e(I I)}=w_{g} \int \mathrm{d} k(|g\rangle\langle k|+| k\rangle\langle g|)$.

Let us project equation (A.1) onto $|k\rangle$ :

$$
\begin{aligned}
\left\langle k\left|H_{2 e}\right| \phi_{\eta}^{e}\right\rangle & =\left\langle k\left|H_{e(0)}\right| \phi_{\eta}^{e}\right\rangle+\left\langle k\left|H_{2 e(I)}\right| \phi_{\eta}^{e}\right\rangle+\left\langle k\left|H_{2 e(I I)}\right| \phi_{\eta}^{e}\right\rangle \\
& =\epsilon_{k}\left\langle k \mid \phi_{\eta}^{e}\right\rangle+w_{e}\left\langle e \mid \phi_{\eta}^{e}\right\rangle+w_{g}\left\langle g \mid \phi_{\eta}^{e}\right\rangle=\epsilon_{\eta}^{e}\left\langle k \mid \phi_{\eta}^{e}\right\rangle .
\end{aligned}
$$

We can do the same with the discrete states $|g\rangle$ and $|e\rangle$ :

$$
\begin{aligned}
\left\langle g\left|H_{2 e}\right| \phi_{\eta}^{e}\right\rangle & =\left\langle g\left|H_{e(0)}\right| \phi_{\eta}^{e}\right\rangle+\left\langle g\left|H_{2 e(I)}\right| \phi_{\eta}^{e}\right\rangle+\left\langle g\left|H_{2 e(I I)}\right| \phi_{\eta}^{e}\right\rangle \\
& =\epsilon_{g}\left\langle g \mid \phi_{\eta}^{e}\right\rangle+w_{g} \int \mathrm{d} k\left\langle k \mid \phi_{\eta}^{e}\right\rangle=\epsilon_{\eta}^{e}\left\langle g \mid \phi_{\eta}^{e}\right\rangle \\
\left\langle e\left|H_{2 e}\right| \phi_{\eta}^{e}\right\rangle & =\left\langle e\left|H_{e(0)}\right| \phi_{\eta}^{e}\right\rangle+\left\langle e\left|H_{2 e(I)}\right| \phi_{\eta}^{e}\right\rangle+\left\langle e\left|H_{2 e(I I)}\right| \phi_{\eta}^{e}\right\rangle \\
& =\epsilon_{e}\left\langle e \mid \phi_{\eta}^{e}\right\rangle+w_{e} \int \mathrm{d} k\left\langle k \mid \phi_{\eta}^{e}\right\rangle=\epsilon_{\eta}^{e}\left\langle e \mid \phi_{\eta}^{e}\right\rangle
\end{aligned}
$$

From the eigenvector's normalization, we can also get the following expression

$$
\left|\left\langle g \mid \phi_{\eta}^{e}\right\rangle\right|^{2}+\left|\left\langle e \mid \phi_{\eta}^{e}\right\rangle\right|^{2}+\int \mathrm{d} k\left|\left\langle k \mid \phi_{\eta}^{e}\right\rangle\right|^{2}=1 .
$$


Defining the fundamental energy level as $\epsilon_{g}=0$, and using Eq. (A.3a) and (A.3b) we can write

$$
\left\langle e \mid \phi_{\eta}^{e}\right\rangle=\frac{\epsilon_{\eta}^{e} w_{e}}{w_{g}\left(\epsilon_{\eta}^{e}-\epsilon_{e}\right)}\left\langle g \mid \phi_{\eta}^{e}\right\rangle
$$

From Eq. (A.2), we have

$$
\left\langle k \mid \phi_{\eta}^{e}\right\rangle=\frac{1}{\epsilon_{\eta}^{e}-\epsilon_{k}}\left(w_{g}+\frac{\epsilon_{\eta}^{e} w_{e}^{2}}{w_{g}\left(\epsilon_{\eta}^{e}-\epsilon_{e}\right)}\right)\left\langle g \mid \phi_{\eta}^{e}\right\rangle,
$$

and from the normalization condition, we obtain

$$
\left\langle g \mid \phi_{\eta}^{e}\right\rangle=\left\{\frac{1}{1+\left[\frac{\epsilon_{\eta}^{e} w_{e}}{w_{g}\left(\epsilon_{\eta}^{e}-\epsilon_{e}\right)}\right]^{2}+\left[w_{g}+\frac{\epsilon_{\eta}^{e} w_{e}^{2}}{w_{g}\left(\epsilon_{\eta}^{e}-\epsilon_{e}\right)}\right]^{2} \int \mathrm{d} k\left(\frac{1}{\epsilon_{\eta}^{e}-\epsilon_{k}}\right)^{2}}\right\}^{1 / 2} .
$$

Therefore, Eq. (A.5), (A.6) and (A.7) give us the explicit form of the three coeficientes.

[1] L. Davidovich, M. Brune, J. M. Raimond, and S. Haroche, Phys. Rev. A 53, 1295 (1996).

[2] M. Brune, E. Hagley, J. Dreyer, X. Maître, A. Maali, C. Wunderlich, J. M. Raimond, and S. Haroche, Phys. Rev. Lett. 77, 4887 (1996).

[3] Y. Yamamoto, S. Machida and O. Nilsson, Phys. Rev. A 34, 4025 (1986).

[4] J. Bergou, L. Davidovich, M. Orszag, C. Benkert, M. Hillery, and M. O. Scully, Phys. Rev. A 40, 5073 (1989).

[5] M. A. M. Marte and P. Zoller, Phys. Rev. A 40, 5774 (1989).

[6] P. Meystre, Opt. Lett. 12, 669 (1987).

[7] P. Meystre and E. M. Wright, Phys. Rev. A 37, 2524 (1988).

[8] G. Rempe and H. Walther, Phys. Rev. A 42, 1650 (1990).

[9] H. Paul and Th. Richter, Opt. Commun. 85, 508 (1991).

[10] H.-J. Briegel, B.-G. Englert, N. Sterpi, and H. Walther, Phys. Rev. A 49, 2962 (1994).

[11] C. Cohen-Tannoudji, J. Dupont-Roc, G. Grynberg, Atom-Photon Interactions: Basic Processes and Applications (Wiley, New York, 1992). 